\title{
Influence of Even-Order Dispersion on Super-Sech Soliton Transmission Quality under Coherent Crosstalk
}

\author{
Aleksandra Panajotovic,, ${ }^{1}$ Daniela Milovic, ${ }^{1}$ Anjan Biswas, ${ }^{2}$ and Essaid Zerrad ${ }^{3}$ \\ ${ }^{1}$ Department of Telecommunications, Faculty of Electronic Engineering, University of Niš, Aleksandra Medvedeva 14, \\ $18000 \mathrm{Niš}$, Serbia \\ ${ }^{2}$ Department of Applied Mathematics and Theoretical Physics, Center for Research and Education in Optical Sciences and Applications, \\ Delaware State University, 1200 N DuPont Highway, Dover, DE 19901, USA \\ ${ }^{3}$ Department of Physics and Pre-Engineering, Delaware State University, 1200 N DuPont Highway, Dover, DE 19901, USA
}

Correspondence should be addressed to Anjan Biswas, biswas.anjan@gmail.com

Received 14 February 2008; Accepted 15 April 2008

Recommended by Gang-Ding Peng

The transmission speed of optical network strongly depends on the impact of higher order dispersion. In presence of coherent crosstalk, which cannot be otherwise controlled by optical filtering, the impact of higher order dispersions becomes more pronounced. In this paper, the general expressions, that describe pulse deformation due to second- and fourth-order dispersions in a single-mode fiber, are given. The responses for such even-order dispersions, in presence of coherent crosstalk, are characterized by waveforms with long trailing edges. The transmission quality of optical pulses, due to both individual and combined influence of second- and fourth-order dispersions, is studied in this paper. Finally, the pulse shape and eye diagrams are obtained.

Copyright (c) 2008 Aleksandra Panajotovic et al. This is an open access article distributed under the Creative Commons Attribution License, which permits unrestricted use, distribution, and reproduction in any medium, provided the original work is properly cited.

\section{Introduction}

Intramodal or chromatic dispersion is a major limiting factor in ultrahigh-speed optical transmission and may occur in all types of optical fibers. It originates from the finite spectral width of the optical source [1-5] and causes broadening of each transmitted mode ( $\mathrm{LP}_{01}$ in single-mode fiber), and thus induces an effect that is known as intersymbol interference (ISI). At high transmission speed, higher order dispersion becomes a severe limiting factor and must thus be taken into consideration.

The strong harmful effect of second-order dispersion is well known in literature and linear commercial systems use dispersion shifted fibers with minimal second-order dispersion in the 1.55-micrometer region [1]. Investigation of new methods and new technologies that could reduce and minimize degrading influence of the second-order dispersion was of interest in the past decade. Different techniques may extend the transmission distance of a dispersionlimited system by a factor of two or so but are unsuitable for long-haul systems for which group velocity dispersion (GVD) has to be compensated along the transmission line in a periodic fashion. Some of the techniques are dispersion-shifted fiber (DSF), dispersion-flattened fiber (DFF), dispersion-compensating fiber (DCF), dispersionmanaged optical fiber, temporal optical conjugation (TOC), and so forth. Chromatic dispersion in optical fibers can add additional noise through phenomena such as intersymbol interference and mode-partition noise.

In the optical transmission system with bit rate higher than hundreds of $\mathrm{Mb} / \mathrm{s}$, the influence of fourth-order dispersion on pulse propagation must not be neglected $[1,2]$. In some cases, it is necessary to investigate the combined effect of second- and fourth-order dispersions on transmission quality of signal. In this paper, we investigate the supersech optical soliton transmission under the influence of such even-order dispersions assuming that odd-order dispersion is fully compensated. Soliton transmission systems can potentially provide exceptionally high information transmission capacity over long distances. Unfortunately for ultralong distance systems, however, the maximum information bit rate depends strongly on the dispersion and intersymbol interference.

Another limiting factor in optical communication systems is optical crosstalk. It represents leakage from other 
optical signal to useful optical signal. The effects of optical crosstalk were first studied by Moura et al. in 1997 [5]. Two types of crosstalk can occur, which depends on whether the crosstalk channel is a different wavelength to the signal (incoherent or out-of-band) or at the same wavelength (coherent or in-band crosstalk). Incoherent crosstalk can in most cases be kept under control with optical filtering at the receiver. Coherent crosstalk [3] is far more detrimental than incoherent crosstalk because it cannot be controlled in this way and thus it influences optical pulse propagation along the optical fiber under chromatic dispersion (secondor fourth-order).This paper clarifies optical pulse deformation caused by even-order dispersion. First, the intrinsic fiber impulsive response for the higher order dispersion is expressed in general terms. Next, the impulsive response of the linear fiber to an ultrashort pulse corrupted by coherent crosstalk is given. Finally, numerically obtained results show the impact of coherent crosstalk on system transmission quality by eye diagrams.

\section{Theory}

Ultrashort pulses from lasers often have a temporal shape that can be analytically modeled by general form of supersech function given by

$$
s(t)=\sqrt{P_{0}} \operatorname{sech}^{m}\left(\frac{t}{T_{0}}\right) \cdot e^{j \omega_{0} t},
$$

where $\omega_{0}$ is optical carrier frequency, $P_{0}$ is optical pulse peak power, and $T_{0}$ is pulse width, and $m$ represents the parameter that determines the super-sech pulse shape. This is mathematical model that is found to be most appropriate to describe laser-induced ultrashort optical pulse shape.

From the original point of view, coherent crosstalk is at the same frequency as useful signal but is often time and phase shifted from the useful optical signal. Taking this fact into consideration, the model of coherent crosstalk can be described as

$$
s_{i}(t)=\sqrt{P_{i}} \operatorname{sech}^{m}\left(\frac{t}{T_{0}}-b^{\prime}\right) \cdot e^{j\left(\omega_{0} t+\phi\right)}
$$

where $P_{i}$ is crosstalk signal peak power and $b\left(b=b^{\prime} T_{0}\right)$ is crosstalk time shift. The value of time shift (propagation delay) depends on the nature of coherent crosstalk (inband crosstalk or multipath reflection) $[3,4]$. The phase shift $\varphi$ varies in random way due to temperature variation and wavelength variation in the range of $[0, \pi]$.

Coherent crosstalk influence on the initial optical pulse is equal to a linear superposition of useful optical pulse and crosstalk. The linear superposition is due to the fact that coherent crosstalk is at the same frequency as useful optical signal. Hence, the resulting pulse $s_{r}(t)$ that will propagate through linear fiber can be expressed completely by its envelope and phase as

$$
\begin{aligned}
& \left|s_{r}(t)\right|=\sqrt{Q}, \\
& \psi(t)=\operatorname{arctg}\left[\frac{\sqrt{P_{i}} \operatorname{sech}^{m}\left(t / T_{0}-b^{\prime}\right) \sin \phi}{\sqrt{P_{0}} \operatorname{sech}^{m}\left(t / T_{0}\right)+\sqrt{P_{i}} \operatorname{sech}^{m}\left(t / T_{0}-b^{\prime}\right) \cos \phi}\right],
\end{aligned}
$$

where $Q$ denotes $\left(\sqrt{P_{0}} \operatorname{sech}^{m}\left(t / T_{0}\right)\right)^{2}+2 \sqrt{P_{0}} \operatorname{sech}^{m}\left(t / T_{0}\right)$. $\sqrt{P_{i}} \operatorname{sech}^{m}\left(t / T_{0}-b^{\prime}\right) \cos \phi+\left(\sqrt{P_{i}} \operatorname{sech}^{m}\left(t / T_{0}-b^{\prime}\right)\right)^{2}$.

For an arbitrary input optical pulse, a general expression of fiber response $r(t, L)$ is given in the literature [1] for an arbitrary input pulse as

$$
\begin{aligned}
r(t, L)= & \frac{1}{2 \pi} e^{-\alpha L} e^{j\left(\omega_{0} t-\beta_{0} L\right)} \\
& \times \int_{-\infty}^{\infty} F(\omega) e^{j\left(\omega t-\omega \beta_{1} L-(1 / 2 !) \beta_{2} L \omega^{2}-(1 / 3 !) \beta_{3} L \omega^{3}-\cdots\right)} d \omega,
\end{aligned}
$$

where $\beta_{i}(i=1,2, \ldots)$ represents $i$ th order dispersion, $\alpha$ is attenuation constant, and $L$ is propagation distance. $F(\omega)$ is Fourier transform of input pulse and for even values of exponent $m$ is obtained in terms of Gauss' hypergeometric function as

$$
\begin{array}{r}
F(\omega)=2^{m} T_{0}\left(\frac{{ }_{2} F_{1}\left(m,\left(m-i T_{0} \omega\right) / 2,1+\left(m-i T_{0} \omega\right) / 2,-1\right)}{m-i T_{0} \omega}\right. \\
\left.+\frac{{ }_{2} F_{1}\left(m,\left(m+i T_{0} \omega\right) / 2,1+\left(m+i T_{0} \omega\right) / 2,-1\right)}{m+i T_{0} \omega}\right),
\end{array}
$$

where the Gauss' hypergeometric function is defined as

$$
{ }_{2} F_{1}(a, b ; c ; z)=\sum_{k=0}^{\infty} \frac{(a)_{k}(b)_{k}}{(c)_{k}} \frac{z^{k}}{k !} .
$$

The Fourier transform of input optical pulse for odd $m$ values is

$$
\begin{aligned}
F(\omega)= & \frac{1}{\sqrt{\pi}} 2^{m / 2-2} e^{-(1 / 4) \pi((k-1) i+2 \omega)} \\
& \times\left(B_{-1}\left(\frac{1}{4}(k-1-2 i \omega), \frac{1}{2}(3-k)\right)\right. \\
& \left.\quad+e^{\pi \omega} B_{-1}\left(\frac{1}{4}(k-1+2 i \omega), \frac{1}{2}(3-k)\right)\right),
\end{aligned}
$$

where

$$
B_{z}(a, b)=\int_{0}^{z} t^{a-1}(1-t)^{b-1} d t
$$

is the incomplete beta function. Both hypergeometric functions and incomplete beta functions can be numerically evaluated with any arbitrary precision.

Depending on the crosstalk phase shift value, coherent crosstalk can be constructive or destructive. Let us investigate 
the worst case crosstalk influence, that is, the destructive coherent crosstalk assuming that it appears at the beginning of the optical fiber (e.g., double reflection or in-band crosstalk resulting of WDM components used for routing and switching along the optical network [3]). The received pulse shape under above mentioned crosstalk influence and under the influence of second- or fourth-order dispersion is

$$
\begin{gathered}
r_{2,4}(t, L)=\frac{\sqrt{P_{0}} T_{0}}{2} e^{-\alpha L} e^{j\left(\omega_{0} t-\beta_{0} L+\theta(t)\right)} \sqrt{I_{1}^{2}(t)+I_{2}^{2}(t)}, \\
\theta(\tau)=\operatorname{arctg} \frac{I_{2}(\tau)}{I_{1}(\tau)}
\end{gathered}
$$

with

$$
\begin{aligned}
I_{1}(t)= & \int_{-\infty}^{\infty} \operatorname{sech}^{m}\left(\frac{\pi T_{0} \omega}{2}\right) \\
& \times\left[\left(1-\sqrt{\frac{P_{i}}{P_{0}}} \cos \left(b^{\prime} T_{0} \omega\right)\right) \cos \left(\omega t-b_{2} \omega^{2}-b_{4} \omega^{4}\right)\right. \\
& \left.\quad-\sqrt{\frac{P_{i}}{P_{0}}} \sin \left(b^{\prime} T_{0} \omega\right) \sin \left(\omega t-b_{2} \omega^{2}-b_{4} \omega^{4}\right)\right] d \omega, \\
I_{2}(t)= & \int_{-\infty}^{\infty} \operatorname{sech}^{m}\left(\frac{\pi T_{0} \omega}{2}\right) \\
& \times\left[\left(1-\sqrt{\frac{P_{i}}{P_{0}}} \cos \left(b^{\prime} T_{0} \omega\right)\right) \sin \left(\omega t-b_{2} \omega^{2}-b_{4} \omega^{4}\right)\right. \\
& \left.\quad+\sqrt{\frac{P_{i}}{P_{0}}} \sin \left(b^{\prime} T_{0} \omega\right) \cos \left(\omega t-b_{2} \omega^{2}-b_{4} \omega^{4}\right)\right] d \omega,
\end{aligned}
$$

where

$$
b_{n}=\left(\frac{\beta_{n} L}{n !}\right), \quad n=2,4 .
$$

If we consider only the influence of second- and fourth-order dispersion, in (9)-(11) we set $b_{2}=b_{4}=0$.

\section{The Influence of Even-Order Dispersion on Super-Sech Pulse Propagation}

For a binary data sequence with 0 and 1 transmitted along the optical fiber, the digital bit rate (B) must be less than the reciprocal of the broadened pulse duration. This is the main condition for nonoverlapping of light pulses down an optical fiber link. In the following figures (Figures 1, 2, and 3 ) we consider such cases for the super-sech optical pulse and crosstalk given by (1) and (2), respectively, with $m=2$. Depending on the value of crosstalk time shift, coherent crosstalk can be left $(b<0)$ or right $(b>0)$ shifted in regard to center of data in binary sequences. In all the figures in this paper, fiber length is expressed in terms of dispersion length which is for $n$ th-order dispersion that is given by

$$
L_{D}=\frac{\left(T_{0}\right)^{n}}{\left|\beta_{n}\right|} .
$$

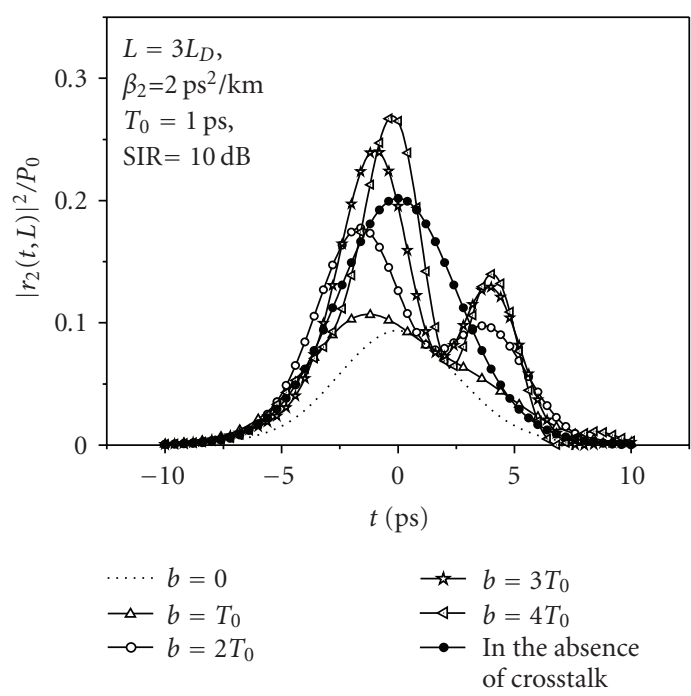

Figure 1: Pulse shape at the end of the optical fiber $\left(L=3 L_{D}\right)$ under second-order dispersion for SIR $=10 \mathrm{~dB}$ and different time shifts $b>0$.

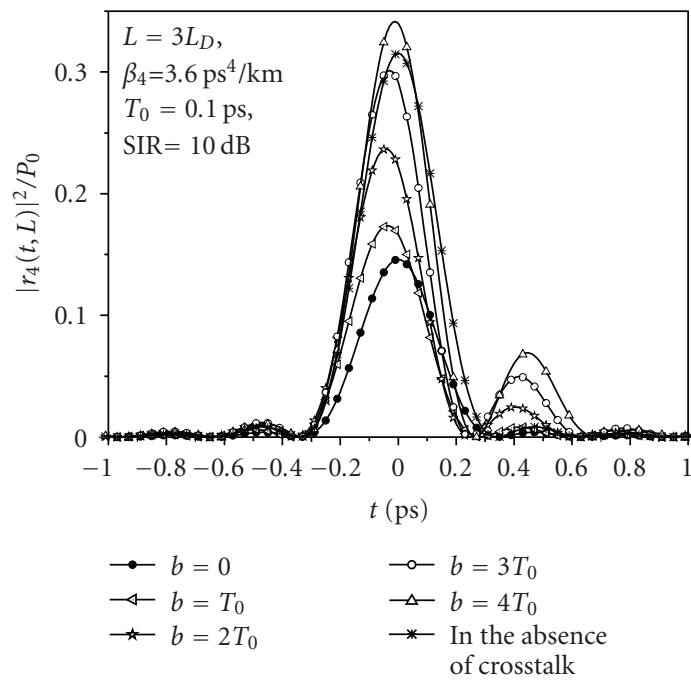

Figure 2: Pulse shape at the end of the optical fiber $\left(L=3 L_{D}\right)$ under fourth-order dispersion for SIR $=10 \mathrm{~dB}$ and different time shifts $b>0$.

Signal-to-interference ratio (SIR) is defined as follows:

$$
\operatorname{SIR}=10 \log \frac{\left|r_{2,4}(t, L)\right|^{2}}{P_{0}}
$$

Second-order dispersion induces asymmetrical broadening of pulse and there is no need to investigate influence of both $b>0$ and $b<0$. Figure 1 shows the strong influence of crosstalk even for $b>T_{0}$. Great time shift of crosstalk induces the asymmetrical pulse deformation and occurrence of trailing edges. Long trailing edges may give rise to intersymbol interference (ISI) and thus limits the transmission length. Because of both, the noisy nature of the input to clock-recovery circuit and noise produced by optical amplifiers, timing jitter can also be produced. Then, 


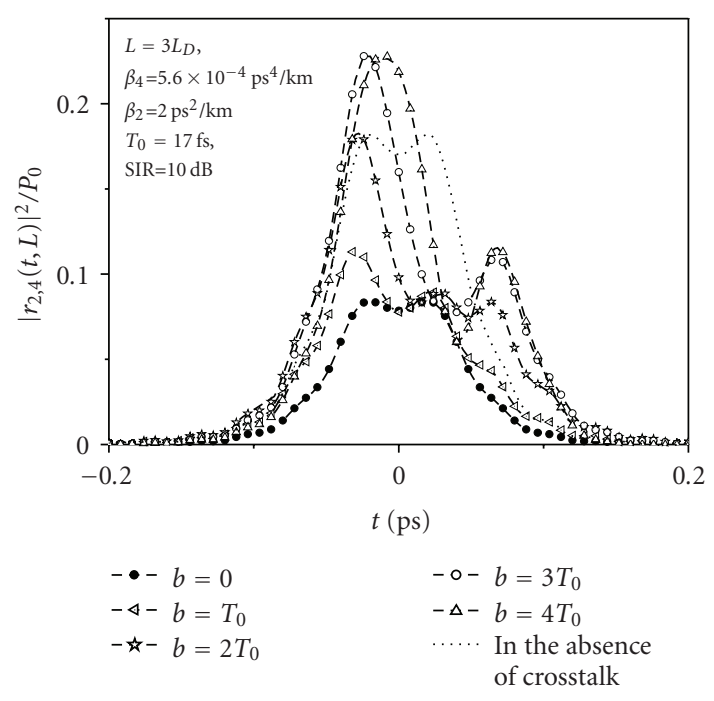

Figure 3: Pulse shape at the end of the optical fiber $\left(L=3 L_{D}\right)$ under second- and fourth-order dispersion for SIR $=10 \mathrm{~dB}$ and different time shifts $b>0$.

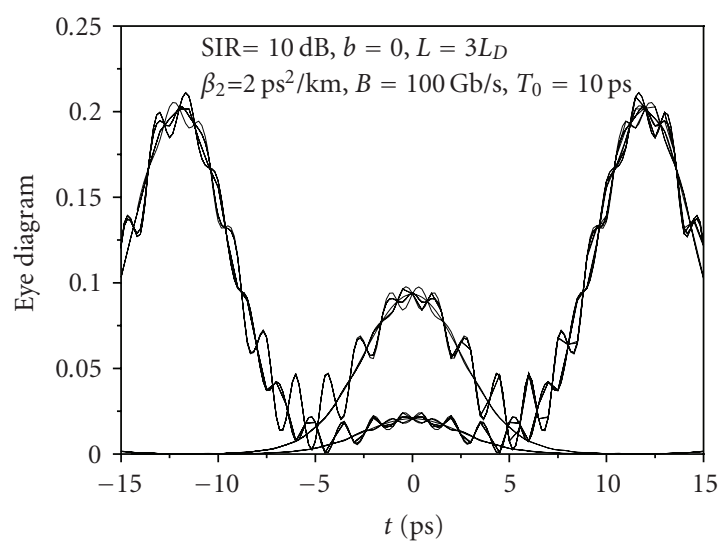

Figure 4: Eye diagram for individual influence of second-order dispersion in the presence of worst case crosstalk.

as previously mentioned, asymmetrical pulse deformation may be dangerous. The worst case in detection process occurs for $b=0$. This situation is very often observed in switching systems. The eye diagram for the worst case is depicted in Figure 4.

There are methods that can compensate for degrading influence of second- and third-order dispersion. In such cases, fourth-order dispersion will have the great influence on pulse shape. Fourth-order dispersion distorts the pulse shape symmetrically around zero and as a result it produces long trailing edges just like for the second-order dispersion. Because of symmetrical deformation of pulse induced by fourth-order dispersion, the greatest error in the detection process will appear for zero crosstalk timing shift. Great absolute values of timing shift increase ISI, if $\mathrm{B}$ is high enough to induce considerable overlapping of pulses. Figure 5 compares eye diagram for both in absence of crosstalk and in the presence of the most destructive

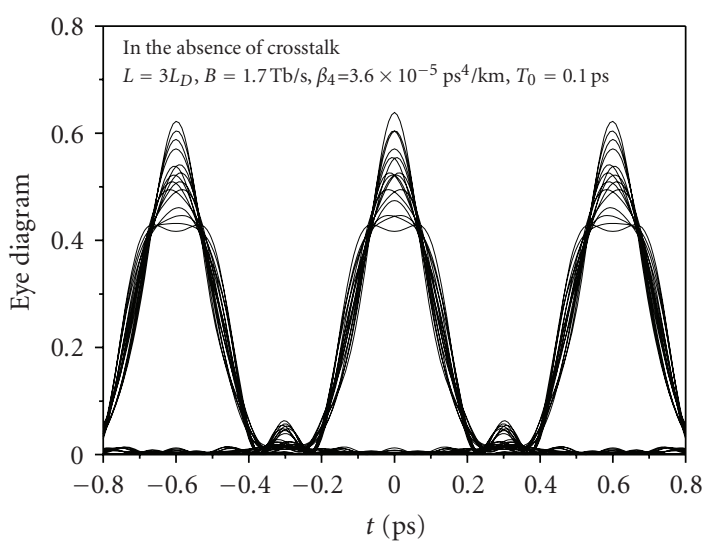

(a)

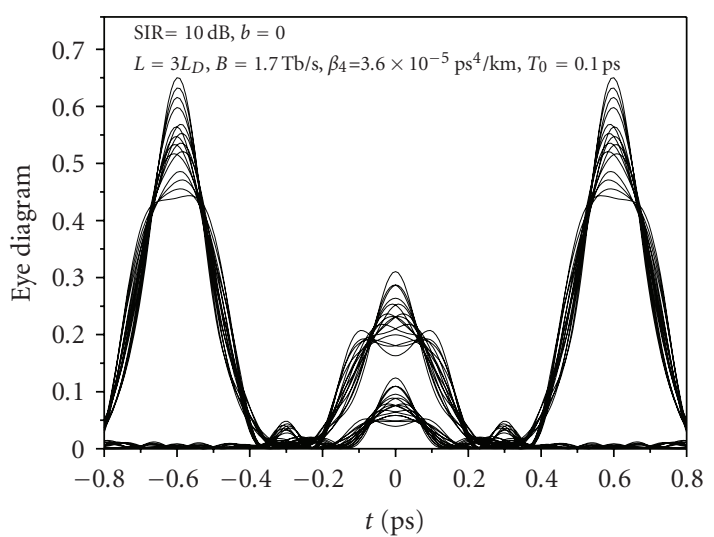

(b)

Figure 5: Eye diagram for individual influence of fourth-order dispersion $\left(L=3 L_{D}\right)$ : (a) in absence of crosstalk, (b) in the presence of worst case crosstalk.

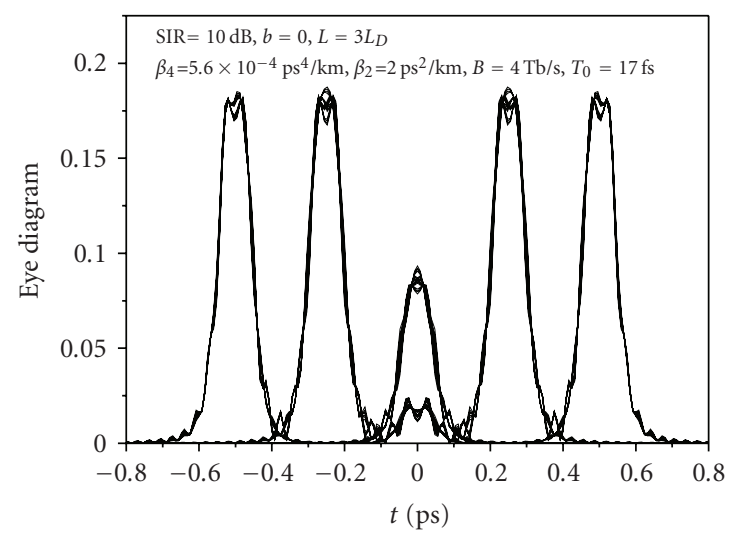

FIGURE 6: Eye diagram for joint influence of second- and fourthorder dispersion $\left(L=3 L_{D}\right)$ in the presence of worst case crosstalk.

crosstalk. It can be seen that eye opening is 10 times decreased in presence of crosstalk which unquestionably leads to bit error rate degradation.

Figures 3 and 6 show joint effect of second- and fourthorder dispersions. Super-sech pulse at the receiver (Figure 3) 
is broadened by second- and fourth-order dispersion interplay and it has a long trailing edge as a result of crosstalk. Eye diagram for this case is shown in Figure 6.

\section{Conclusion}

This paper studied the quality of transmission of supersech solitons, in a single-mode fiber, in presence of secondand fourth-order dispersions. Coherent crosstalk is considered as it is one of the most detrimental effects on the soliton transmission quality and performance enhancement of optical systems. The analytical expressions of the pulse shape, in presence of coherent crosstalk, in optical fibers were derived. Finally, numerical simulations and eye diagrams are obtained.

\section{Acknowledgment}

The research work of Anjan Biswas was fully supported by NSF-CREST Grant no. HRD-0630388 and this support is genuinely and thankfully appreciated.

\section{References}

[1] M. Amemiya, "Pulse broadening due to higher order dispersion and its transmission limit," Journal of Lightwave Technology, vol. 20, no. 4, pp. 591-597, 2002.

[2] F. Ö. Ilday, F. W. Wise, and T. Sosnowski, "High-energy femtosecond stretched-pulse fiber laser with a nonlinear optical loop mirror," Optics Letters, vol. 27, no. 17, pp. 1531-1533, 2002.

[3] E. Iannone, R. Sabella, M. Avattaneo, and G. De Paolis, "Modeling of in-band crosstalk in WDM optical networks," Journal of Lightwave Technology, vol. 17, no. 7, pp. 1135-1141, 1999.

[4] Y. Shen, K. Lu, and W. Gu, "Coherent and incoherent crosstalk in WDM optical networks," Journal of Lightwave Technology, vol. 17, no. 5, pp. 759-764, 1999.

[5] L. Moura, M. Darby, P. M. Lane, and J. J. O’Reilly, "Impact of interferometric noise on the remote delivery of optically generated millimeter-wave signals," IEEE Transactions on Microwave Theory and Techniques, vol. 45, no. 8, part 2, pp. 1398-1402, 1997. 

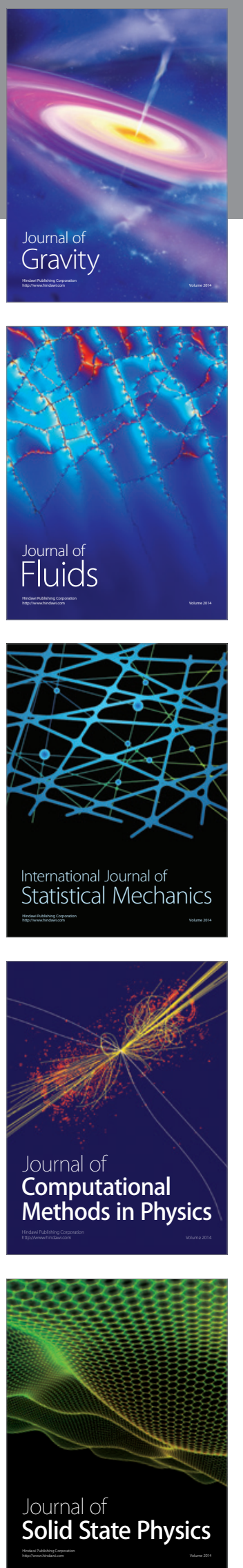

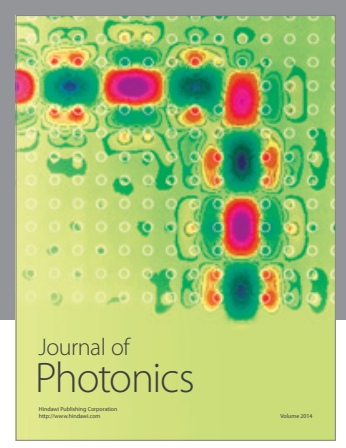

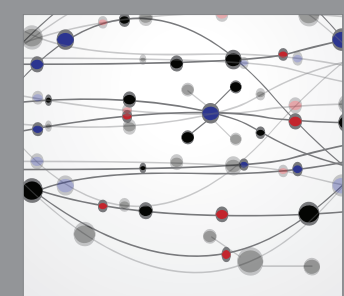

The Scientific World Journal
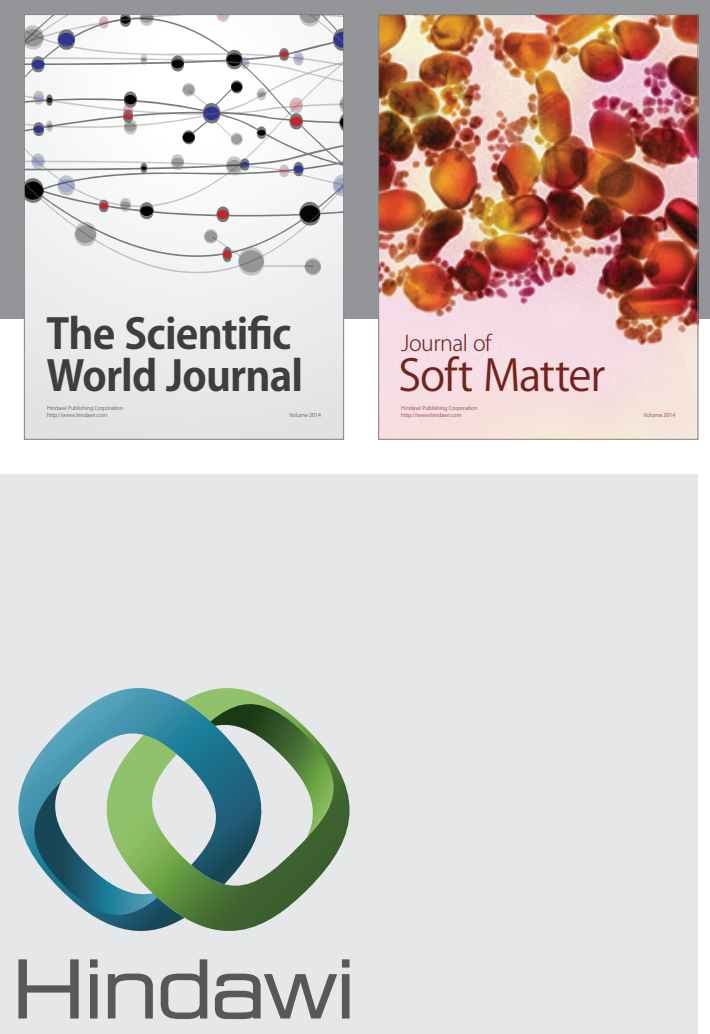

Submit your manuscripts at

http://www.hindawi.com
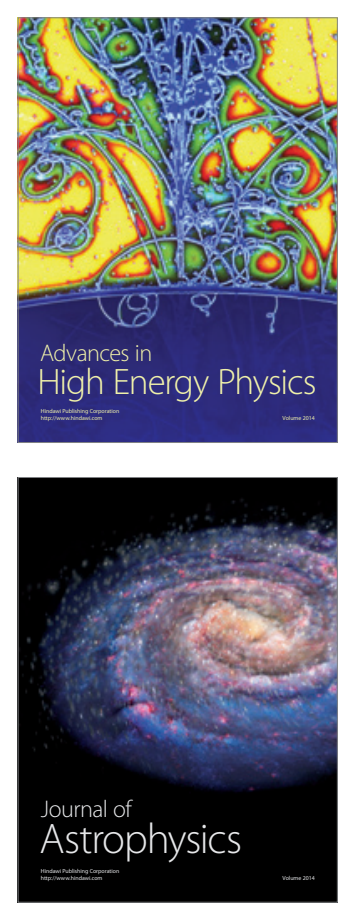
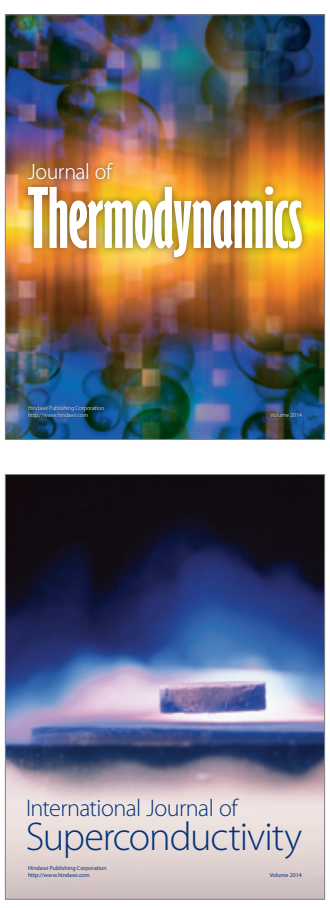
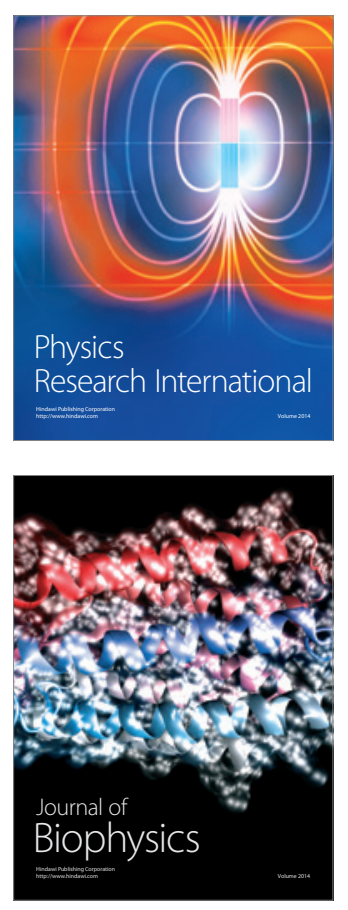
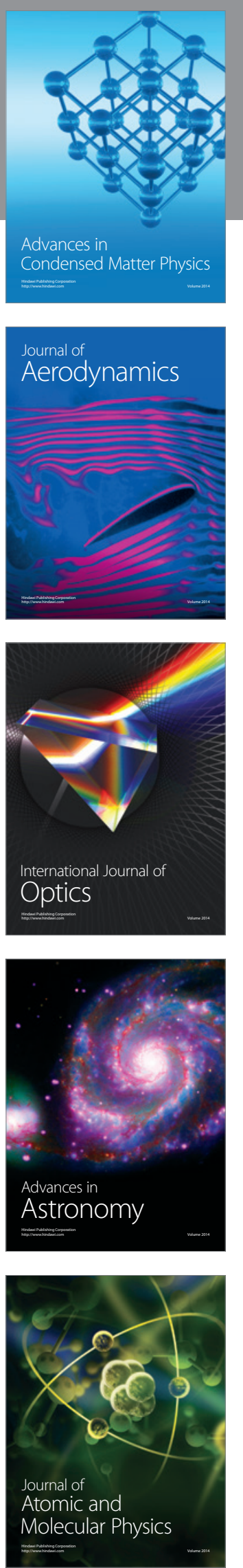